\title{
Effects of Strain Node on the Actuation Performance of Multilayer Cantilevered Piezoactuator with Segmented Electrodes
}

\author{
Quan Bai $\mathbb{D}^{1,2}$ and Xuejun Zheng $\mathbb{D}^{1}$ \\ ${ }^{1}$ School of Mechanical Engineering, Xiangtan 411105, China \\ ${ }^{2}$ Hunan Provincial Key Laboratory of Vehicle Power and Transmission System, Hunan Institute of Engineering, \\ Xiangtan 411101, China \\ Correspondence should be addressed to Xuejun Zheng; zhengxuejun@xtu.edu.cn
}

Received 10 December 2021; Accepted 15 January 2022; Published 3 March 2022

Academic Editor: Jiaqiang E

Copyright (c) 2022 Quan Bai and Xuejun Zheng. This is an open access article distributed under the Creative Commons Attribution License, which permits unrestricted use, distribution, and reproduction in any medium, provided the original work is properly cited.

\begin{abstract}
A model of a segmented electrode multilayer cantilever piezoelectric actuator was established to predict its actuation performance, and then, theoretical and numerical analyses of the strain nodes were performed based on normalized deflection and strain distributions. The segmented electrodes instead of the continuous electrodes are applied in a multilayer cantilever piezoelectric actuator which can avoid the modal displacement offsets at the high vibration modes, thereby enhancing the tip deflection. The theoretical analysis and simulation results show that the tip deflection of the segmented electrode at the second mode was almost $100 \%$ larger than that of the continuous electrode. At the second mode, the maximum error between the theoretical calculation value of the tip deflection and the simulation result is $6.8 \%$. It is because the segmented electrode is optimally designed at the strain node, which avoids the modal displacement offsets of a multilayer cantilever piezoelectric actuator at the high vibration modes; meanwhile, the theoretical results are closer to the FEM simulation results. It reveals that the tip deflection of a multilayer cantilever piezoelectric actuator can be precisely estimated by the proposed model. This research can provide some useful guidance improving the actuation performance and optimizing the design of a multilayer cantilever piezoelectric actuator.
\end{abstract}

\section{Introduction}

Piezoactuators are widely used in microelectromechanical systems (MEMS) because of the characteristics of small size, thinness, and high displacement, such as atomic force microscopes [1], biosensors [2], microelectromechanical switches [3], and micropositioning platforms [4], etc. The actuation performance improvement and design optimization of such devices have always been the main focus of many researchers. In particular, there are many reports in the theoretical research of piezoelectric actuators. Based on the Euler model, Wang et al. [5] and Zhang et al. [6] presented the governing equations for the piezoelectric actuators with a sandwich layer. In a study conducted by Zhang et al. [7], a simple MCPAs distributed parameter model is developed to simulate the fundamental wave of piezoelectricity in thickness-extension mode. In order to reduce the poor piezoelectric effect caused by the damage of piezoelectric materials, some researchers have designed multilayer piezoelectric actuators to improve the flexibility and compactness of the structure. Afonin [8] constructed a generalized structural parameter model of nanomechatronics multilayer electromagnetic elastic actuators. Shivashankar and Gopalakrishnan [9] reported a d33 mode surface-bondable multilayer actuator that can provide large braking force and stroke for driving large, thick, and stiffer structures. Peng et al. [10] proposed a piezoelectric multilayer actuator considering buffer layers and analyzed the dependence of the resonance frequency at the first mode and tip deflection on different layer thicknesses (buffer layer, electrode layer, and substrate layer). The contributions of the above research mainly focused on the first mode while 
ignoring the other higher-frequency modes, because the tip deflection of the cantilever beam at higher modes is smaller than the tip deflection at the first mode. However, piezoelectric cantilever beams can also provide superior performance at higher modes [11], which is more common in energy harvesters [12]. Ly et al. developed a 31-effect piezoelectric bending cantilever based on the Euler-Bernoulli beam theory. Their results indicated that the voltage and bandwidth at the second mode of resonant frequency were much larger than those at the first mode [13]. Except for the first mode, the cantilevered piezoelectric energy harvester (PEH) has fixed strain nodes in other vibration modes, and there are dynamic strain distributions with opposite strain signs on both sides of the node. Theoretical and experimental results demonstrate that covering the strained nodes with continuous electrodes may lead to a strong cancelation of the electrical output [14]. In order to improve the piezoelectric performance, Zizys et al. investigated the segmentation of a vibration-shock cantilevered PEH working in higher transverse vibration modes [15]. Rafique et al. used segmented electrodes to enhance the output power of the $\mathrm{PEH}$ [16]. Liu et al. employed the first and second bending vibration modes to design a novel bonded-type piezoelectric actuator, which obtained higher power density than previous designs [17]. Although segmented electrodes could be applied to MCPAs at the high vibration modes, there is currently no complete electromechanical equation that can describe MCPA with segmented electrodes (MCPA-S).

In this paper, by optimizing the structure of segmented electrodes, a MCPA with segmented electrodes based on the strain nodes is designed to improve the actuation performance [18]. The strain nodes are determined by the normalized deflection and strain distribution [14]. Based on Euler-Bernoulli beam theory and piezoelectric constitutive equation, a complete electromechanical coupling model is developed for the MCPA-S. Here, the electrodes are connected in series at the first mode and in parallel at the second mode, which is different from those reported in [11]. It can prevent the displacement offset in the electric potential, increase the modal electromechanical coupling term, and improve the applied capacity. In order to understand whether segmented electrodes can eliminate the influence of strain node at higher modes, we have studied the relationship of tip deflection of segmented and continuous electrodes MCPAs with excitation frequency, excitation voltage, and beam length under different modes. In addition, the structural parameters of the MCPAs were optimized by simulating different thicknesses of the substrate, piezoelectric, and buffer layers, as well as the different Young's modulus ratios. The proposed model and prediction results can provide useful guidance for optimizing the construction and efficiency of MCPAs.

\section{Design and Modeling}

2.1. Design. Except for the fundamental modes, the dynamic strain distribution of the cantilevered beam changes direction at fixed strain nodes. The modal actuation capability of a cantilevered beam is closely related to the position of the piezoelectric actuators. To increase the driving force, the use of segmented actuators to control adaptive structures is proposed by avoiding the position of dynamic strain phase changes. At high modes, when the top surface of the entire piezoelectric layer was covered by continuous electrodes, the actuation capability had been significantly reduced. Therefore, we apply electrode segmentation at the nodes to a multilayer actuator (considering buffer and electrode layers), which is different from the traditional sandwich structure. This paper takes the MCPA in the second mode as an example to analyze the actuation capability. There is one strain node in the secondorder mode, and the electrode is cut at the node and divided into two sections of electrodes.

Figure 1 depicts a two-dimensional schematic diagram of the MCPA-S. One end of the multilayer cantilever piezoelectric actuator is attached to the base composed of five different layers from bottom to top: the substrate, the buffer, the second electrode, the piezoelectric layer, and the first electrode as shown in Figure 1(a). The first electrode and the piezoelectric layer are cut at the strained node to form segmented electrodes for the MCPA. The contact between the second electrode and the lower surface of the piezoelectric film is continuous, but the contact between the first electrode and the upper surface of the piezoelectric layer is discontinuous. The polarization direction is reversed after passing through the strain node, and the split position is the strain node position $L_{1}$. The different electrode connections are adopted under the different modes of the cantilever beam. At the first mode, the strain distribution is in the same phase for the MCPA, because there is no strain node [10]. Here, the electrode directions in the $L_{1}$ and $L_{1}-L$ regions are opposite, and the electrode wires are connected in series with the applied voltage, as shown in Figure 1(b). At the second mode, there is a strain node and the strain distributions in $L_{1}$ and $L_{1}-L$ regions are 180 degrees out of phase. The connection of the electrodes is described in Figure 1(c), which is arranged in parallel to prevent modal displacement in the electric potential. And then the applied voltage should be applied on the $L_{1}$ and $L_{1^{-}}$$L$ regions of the cantilever beam to generate the deflection for the MCPA.

$Z_{1}, Z_{2}, Z_{3}$, and $Z_{4}$ depict the vertical coordinates of the bottom-surface of substrate, buffer, piezoelectric layer, and the first electrode, respectively. $Z_{i}$ and $Z_{5}$ indicate the vertical coordinates of the top surface of buffer and the second electrode. The length and width of the cantilever beam are denoted by $L$ and $b$. In Cartesian coordinate system, the $x$ and $z$-axes are consistent with the directions 1 and 3, respectively, and the $z$-axis represents the polarization direction of the piezoelectric layer. The coordinate origin of the $\mathrm{x}-\mathrm{z}$ plane corresponds to the leftmost point of the MCPA. The mid-plane of the substrate is denoted by the dotted line. The neutral plane is located at $z_{0}$ from the mid-plane of the substrate. Moreover, $h$ is used to describe the thickness of each layer, and its subscripts $p, s, i$, and e indicate the piezoelectric, substrate, buffer, and electrodes layers, respectively. The transverse deflection $w(x, t)$ of the MCPA occurs along the $z$-axis and is a function with the $x$ value and time $t$. 


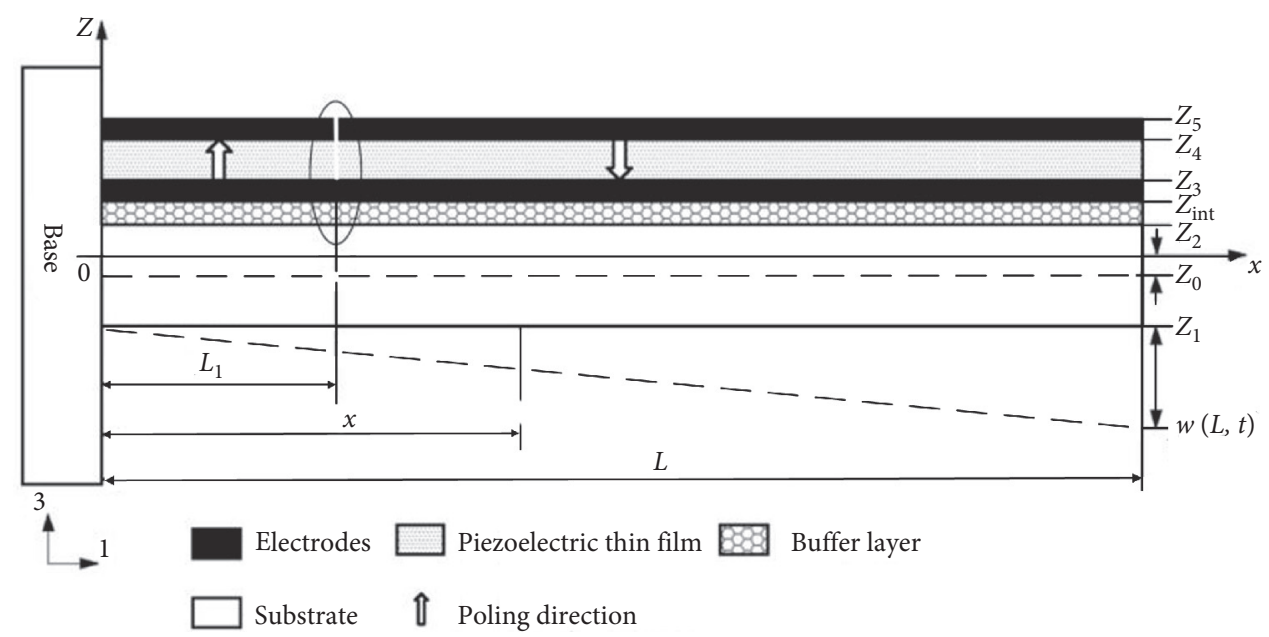

(a)

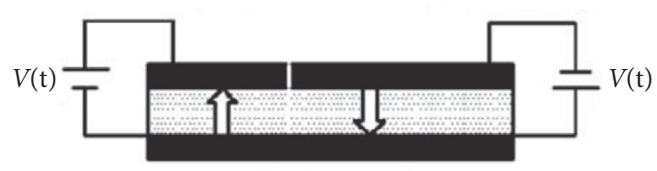

The first mode

(b)

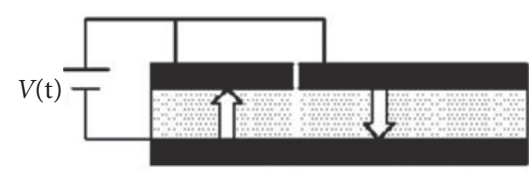

The second mode

(c)

Figure 1: (a) Schematic illustration of the deflection of MCPA-S; (b) electrode wire connection at the first mode (series connection) and (c) at the second mode (parallel connection).

\subsection{Modeling}

2.2.1. Electromechanical Coupling Equation. The constitutive equation for piezoelectric materials can be described in the form [10]

$$
T_{1, p}=c_{11, p} S_{1}-e_{p 31} E_{3},
$$

where stress, strain, and electric field are denoted by $T, S$, and $E$, respectively, elastic constant of the piezoelectric material is described by $c$, and piezoelectric coupling coefficient under steady electric field is depicted by $e_{p}$. Here, the axial strain and polarization direction are marked as subscripts 1 and 3 , respectively. The elastic stiffness component can be calculated by $c_{11, p}=1 / s_{11, p}$ according to the plane-stress presumption of the MCPA. Under a constant electric field, $s_{11, p}$ denotes the elastic compliance. In addition, $e_{31}$ can be expressed as $e_{p 31}=d_{31} / s_{11, p}$ with the commonly used piezoelectric constant $d_{31}$. The constitutive equations applied to the substrate layer and the buffer layer can be given as [19]

$$
\begin{aligned}
& T_{1, s}=c_{11, s} S_{1}, \\
& T_{1, i}=c_{11, s} S_{1} .
\end{aligned}
$$

The constitutive equation for the electrode layer is [19]

$$
T_{1, e}=c_{11, e} S_{1},
$$

and the axial strain $S_{1}$ at value $x$ and time $t$ can be obtained by [20]

$$
S_{1}(x, t)=-z \frac{\partial^{2} w(x, t)}{\partial x^{2}} .
$$

According to our previous research [7], the position of the neutral plane $z_{0}$ can be given by $z_{0}=\left[\left(c_{11, e} h_{e 2}+c_{11, p}\right.\right.$ $\left.h_{p}\right)\left(2 h_{e 1}+2 h_{i}+h_{s}+h_{p}\right)+c_{11, e} h_{e_{2}} \quad\left(h_{e_{2}}+h_{p}\right) c_{11, e} h_{e_{2}}\left(h_{e_{2}}+\right.$ $\left.\left.h_{p}\right)+\left(c_{11, e} h_{e_{1}}+c_{11, i} h_{i}\right)\left(h_{s}+h_{i}\right)+c_{11, e} h_{e_{1}}\left(h_{e_{1}}+h_{i}\right)\right] /\left(c_{11, s} h_{s}\right.$ $\left.+c_{11, i} h_{i}+c_{11, e} h_{e_{1}}+c_{11, e} h_{e_{2}}+c_{11, p} h_{p}\right)$

According to the moment balance equation (the beam's cross section), the bending moment is given by

$$
M(x, t)=-b\left(\int_{z_{1}}^{z_{2}} T_{1, s} z \mathrm{~d} z+\int_{z_{2}}^{z_{\text {int }}} T_{1, i} z \mathrm{~d} z+\int_{z_{\text {int }}}^{z_{3}} T_{1, e_{1}} z \mathrm{~d} z+\int_{z_{3}}^{z_{4}} T_{1, p} z \mathrm{~d} z+\int_{z_{4}}^{z_{5}} T_{1, e_{2}} z \mathrm{~d} z\right) .
$$

At the first mode, the polarization and electric field are consistent along the entire beam length; thus, the uniform electric field $E_{3}(t)$ can be given in terms of voltage $v(t)$ across the piezoelectric layer and the thickness $h_{p}$ as $E_{3}(t)=-v(t) / h_{p}$. At the second mode, the polarization direction and electric field of the two segmented piezoelectric layers are different. Therefore, for the $0-L_{1}$ area (same direction) the electric field can be obtained by $E_{3}(t)=-v(t) / h_{p}$; and for the $L-L_{1}$ area (opposite direction), $E_{3}(t)=v(t) / h_{p}$. 
By substituting equations (1)-(4) into equation (5) and integrating equation (5), it can be further simplified to

$$
M(x, t)=E I \frac{\partial^{2} w(x, t)}{\partial x^{2}}-v(t) \Gamma(x)
$$

EI represents the bending stiffness of the composite structure's cross section, which is expressed by the following equation:

$$
E I=\frac{b}{3}\left[c_{11, s}\left(z_{2}^{3}-z_{1}^{3}\right)+c_{11, i}\left(z_{i}^{3}-z_{2}^{3}\right)+c_{11, e}\left(z_{3}^{3}-z_{i}^{3}\right)+c_{11, p}\left(z_{4}^{3}-z_{3}^{3}\right)+c_{11, e}\left(z_{5}^{3}-z_{4}^{3}\right)\right]
$$

$\Gamma(x)$ represents the spatial distribution of the electric potential and is related to the modes and the structure of the piezoelectric cantilever beam. For the MCPA with continuous electrodes (MCPA-C), $\Gamma(x)$ is expressed as [21]

$$
\Gamma(x)=\vartheta[H(x)-H(x-L)] 0 \leq x \leq L .
$$

For the MCPA with segmented electrodes (Figure 1), the uniform electric fields are applied at different modes by conducting the different electrode lines. The potential spatial distribution can be regarded as the sum of the two electrode regions. At the first mode, the electric field is exerted to the segmented electrodes in series, and the $\Gamma(x)$ can be derived by

$$
\Gamma(x)=\Gamma_{1}(x)+\Gamma_{2}(x)=\vartheta[H(x)+H(x-L)] .
$$

At the second mode, the electric field is exerted to the segmented electrodes in parallel, and the $\Gamma(x)$ can be derived by

$$
\Gamma(x)=\Gamma_{1}(x)+\Gamma_{2}(x)=\vartheta\left[H(x)+H(x-L)-2 H\left(x-L_{1}\right)\right],
$$

where $H(x)$ denotes the Heaviside function and the coupling term $\vartheta$ is given as

$$
\vartheta=\frac{b e_{31}\left(z_{4}^{2}-z_{3}^{2}\right)}{\left(2 h_{p}\right)}
$$

The configurations of the microcantilever conform to the Euler-Bernoulli beam hypothesis, which have been presented in our previous research [10]. Considering viscous air (medium) damping and Kelvin-Voigt (or strain rate) damping, the governing equation of the cantilever beam can be written as [21]

$\frac{\partial^{2} M(x, t)}{\partial x^{2}}+c_{s} I \frac{\partial^{5} w(x, t)}{\partial x^{4} \partial t}+m \frac{\partial^{2} w(x, t)}{\partial t^{2}}+c_{a} \frac{\partial w(x, t)}{\partial t}=0$

Here, the viscous damping coefficient is denoted by $c_{a}$, the inertia moment of the cross section area is described by $I$, the strain rate damping term is expressed as $c_{s} I$, and the mass per unit length of the MCPA is represented by $m$, which is obtained by

$$
m=b\left(p_{s} h_{s}+p_{e} h_{e 1}+p_{p} h_{p}+p_{e} h_{e 2}+p_{i} h_{i}\right)
$$

where the uniform densities of the different layers are $p_{s}(\mathrm{Si}$ substrate), $p_{i}$ (buffer), $p_{\mathrm{e}}$ (electrodes), and $p_{p}$ (piezoelectric), respectively. The mass per unit length of the different layers is $b p_{s}$ (Si substrate), $b p_{i}$ (buffer), $b p_{e 1}$ (electrodes), $b p_{p}$ (piezoelectric), and $b p_{e 2}$ (electrodes), respectively. Finally, by inserting equation (6) into equation (11), the electromechanical coupling equation of the MCPA at the first two modes can be obtained:

$$
E I \frac{\partial^{4} w(x, t)}{\partial x^{4}}+c_{s} I \frac{\partial^{5} w(x, t)}{\partial x^{4} \partial t}+m \frac{\partial^{2} w(x, t)}{\partial t^{2}}+c_{a} \frac{\partial w(x, t)}{\partial t}=v(t) \frac{d^{2} \Gamma(x)}{d x^{2}}
$$

2.2.2. Modal Analysis. Based on the standard modal expansion approach, a series of the absolutely uniformly convergent eigenfunctions are used to describe the transverse deflection of the cantilever beam [11]:

$$
w(x, t)=\sum_{r=1}^{\infty} \phi_{r}(x) \eta_{r}(t)
$$

The mass normalized eigenfunction and modal coordinate of the clamped-free beam at the $r^{\text {th }}$ vibration mode are, respectively, given by $\phi_{r}(x)$ and $\eta_{r}(t)$. The deflection of the cantilever beam can be exactly obtained from this equation. $\phi_{r}(x)$ is written as [11]

$$
\phi_{r}(x)=\sqrt{\frac{1}{m L}}\left[\left(\cosh \frac{\lambda_{r}}{L} x-\cos \frac{\lambda_{r}}{L} x\right)-\sigma_{r}\left(\sinh \frac{\lambda_{r}}{L} x-\sin \frac{\lambda_{r}}{L} x\right)\right],
$$

and it satisfies the orthogonality conditions [9]. 


$$
\begin{gathered}
\int_{0}^{L} m \phi_{r}(x) \phi_{s}(x) \mathrm{d} x=\delta_{r s}, \\
\int_{0}^{L} E I \phi_{s}(x) \frac{\mathrm{d}^{4} \phi_{r}(x)}{\mathrm{d} x^{4}} \mathrm{~d} x=\omega_{r}^{2} \delta_{r s} .
\end{gathered}
$$

Here, $\lambda_{r}$ (dimensionless frequency number) of the $r^{\text {th }}$ vibration mode can be expressed as follows:

$$
1+\cos \lambda \cosh \lambda=0
$$

and $\sigma_{r}$ is given by

$$
\sigma_{r}=\frac{\sinh \lambda_{r}-\sin \lambda_{r}}{\cosh \lambda_{r}+\cos \lambda_{r}}
$$

Bending strain distribution can be measured directly by the curvature eigenfunction that is the second derivative of the displacement eigenfunction (equation (16)). For a positive definite system $\left(\lambda_{r}>0\right)$, the positions of the strain nodes can be determined by calculating the roots of equation (20) at $0<\bar{x}<1$

$$
\left(\cosh \lambda_{r} \bar{x}+\cos \lambda_{r} \bar{x}\right)-\sigma_{r}\left(\sinh \lambda_{r} \bar{x}+\sin \lambda_{r} \bar{x}\right)=0,
$$

where $\bar{x}=x / L$ denotes the length position (dimensionless) for the MCPA. By combining equation (20) with equations (18) and (19), the strain nodes positions (dimensionless) of the first three modes can be obtained in Table 1 .

In addition, $\omega_{r}$ is the undamped natural frequency of the $r^{\text {th }}$ mode, which is written as

$$
\omega_{r}=\frac{\lambda_{r}^{2}}{L^{2}} \sqrt{\frac{E I}{m}} .
$$

Equation (16) is simplified by using the orthogonal condition of equation (17) and then substituted into equation (15). The mechanical motion equation in modal coordinates can be derived as follows:

$$
\frac{\mathrm{d}^{2} \eta_{r}(t)}{\mathrm{d} t^{2}}+2 \xi_{r} \omega_{r} \frac{\mathrm{d} \eta_{r}(t)}{\mathrm{d} t}+\omega_{r}^{2} \eta_{r}(t)-\chi_{r} v(t)=0,
$$

where $\xi_{r}$ denotes the modal mechanical damping ratio. The coupling term (modal electromechanical) is defined as

$$
\chi_{r}=\int_{0}^{L} \phi_{r}(x) \frac{\mathrm{d} \Gamma(x)}{\mathrm{d} x^{2}} \mathrm{~d} x .
$$

(23) can be further rewritten as [11]

$$
\begin{aligned}
& \chi_{r}=\vartheta \int_{0}^{L} \phi_{r}(x)\left(\frac{\mathrm{d} \delta(x)}{\mathrm{d} x} \mathrm{~d} x\right), \\
& \chi_{r}=\vartheta \int_{0}^{L} \frac{\mathrm{d} \phi_{r}(x)}{\mathrm{d} x} \delta(x) \mathrm{d} x,
\end{aligned}
$$

where $\delta(x)$ is the Dirac function.

By substituting equation (8) into equation (23), $\chi_{r}$ for the continuous electrodes can be rewritten as

$$
\chi_{r}=\vartheta\left(\left.\frac{\mathrm{d} \phi_{r}(x)}{\mathrm{d} x}\right|_{x=L}\right) \text {. }
$$

For the segmented electrodes, $\chi_{r}$ is related to the spatial distribution of the electric potential at the vibration modes.
By substituting equation (9) into equation (25), $\chi_{r}$ at the first mode can be expressed as

$$
\chi_{1}=\vartheta\left(\left.\frac{\mathrm{d} \phi_{r}(x)}{\mathrm{d} x}\right|_{x=L}\right) .
$$

At the second mode, it can be obtained by

$$
\chi_{2}=\vartheta\left(\left.2 \frac{\mathrm{d} \phi_{2}(x)}{\mathrm{d} x}\right|_{x=L_{1}}-\left.\frac{\mathrm{d} \phi_{2}(x)}{\mathrm{d} x}\right|_{x=L}\right) .
$$

Employing the separating variables method, we record $\eta_{r}(t)=N_{r} e^{j \omega t}$ and $v(t)=V e^{j \omega t}$, where $N_{r}$ and $V$ represent the amplitudes. By substituting them into equation (22), $\eta_{\mathrm{r}}$ can be calculated by

$$
\eta_{r}(t)=\sum_{r=1}^{\infty} \frac{\chi_{r} \phi_{r} V e^{j \omega t}}{\omega_{r}^{2}-\omega^{2}+j 2 \xi_{r} \omega_{r} \omega} .
$$

By substituting equation (28) into equation (15), the transverse deflection can be redescribed as the following formula:

$$
w(x, t)=\sum_{r=1}^{\infty} \frac{\chi_{r} \phi_{r}(x) V e^{j w t}}{\omega_{r}^{2}-\omega^{2}+j 2 \xi_{r} \omega_{r} \omega} .
$$

Finally, the tip deflection that occurred at the free end of the cantilever is expressed as

$$
w(L, t)=\sum_{r=1}^{\infty} \frac{\chi_{r} \phi_{r}(L) V e^{j \omega t}}{\omega_{r}^{2}-\omega^{2}+j 2 \xi_{r} \omega_{r} \omega} .
$$

\section{Verification by FEM Simulation}

3.1. Material Properties and Structural Parameters. The MCPA finite element model consists of $\mathrm{Si}$ substrate, $\mathrm{SiO}_{2}$ buffer, Pt first electrode, piezoelectric, and Pt second electrode. The geometrical dimensions of the segmented electrode MCPA include length $L$, width $b$, thicknesses $h_{s}, h_{i}, h_{e 1}$, $h_{p}, h_{e 2}$, and segmented length $L_{1}$. All dimensions are listed in Table 2. At the first and second mode, the mechanical damping ratios were $\xi_{1}=0.01$ and $\xi_{2}=0.013$, respectively. Poisson's ratio $v=0.3$ was set in this paper, and other material property parameters are shown in Tables 3 and 4 .

3.2. Strain Distribution of the Cantilevers with MCPA. In the simulations, the piezoelectric material is modeled by "solid5" composed of 3D 8-node hexahedral coupled-field elements, and the nonpiezoelectric materials are modeled by "solid 45 " including 8-node linear structural elements. At the beam's fixed end, the freedom degree of displacement is limited to be zero. The electrode connection of the first and second electrodes is implemented using coupling commands. For the upper surface of the second electrode layer and the lower surface of the piezoelectric layer, the voltage is coupled and constrained to be zero. The applied voltage is coupled to the upper surface of the first electrode layer. Figures 2(a), 2(b), 3(a) and 3(b) show the raw and fine mesh of the finite element (FEM); the circled area marks the position of the strain node. The strain distributions of the continuous and segmented 
TABle 1: Frequencies and strain node positions (dimensionless) of a cantilevered beam for the first three modes.

\begin{tabular}{lccc}
\hline Mode & $\lambda_{r}$ & \multicolumn{2}{c}{$\begin{array}{c}\text { Strain node positions on } \\
\text { the } x \text {-axis }(\bar{x}=x / L)\end{array}$} \\
\hline 1 & 1.87510407 & - & - \\
2 & 4.69409113 & 0.2165 & - \\
3 & 7.85475744 & 0.1323 & 0.4965 \\
\hline
\end{tabular}

TABle 2: The geometrical dimensions.

\begin{tabular}{lccccccc}
\hline$b$ & $\begin{array}{c}L \\
(\mu \mathrm{m})\end{array}$ & $\begin{array}{c}L_{1} \\
(\mu \mathrm{m})\end{array}$ & $\begin{array}{c}h_{s} \\
(\mu \mathrm{m})\end{array}$ & $\begin{array}{c}h_{i} \\
(\mu \mathrm{m})\end{array}$ & $\begin{array}{c}h_{\mathrm{e} 1} \\
(\mu \mathrm{m})\end{array}$ & $\begin{array}{c}h_{p} \\
(\mu \mathrm{m})\end{array}$ & $\begin{array}{c}h_{e 2} \\
(\mu \mathrm{m})\end{array}$ \\
\hline 200 & 1000 & 216 & 20 & 1 & 1 & 2 & 1 \\
\hline
\end{tabular}

TABLE 3: Mechanical properties of the different materials.

\begin{tabular}{lcccc}
\hline Material & $\mathrm{Si}$ & $\mathrm{SiO}_{2}$ & $\mathrm{Pt}$ & Piezoelectric \\
\hline Density $(\mathrm{kg} / \mathrm{m} 3)$ & 2330 & 2200 & 21400 & 7550 \\
Young's modulus $(\mathrm{GPa})$ & 170 & 72 & 168 & 81 \\
\hline
\end{tabular}

electrode MCPAs at the first two modes are, respectively, presented in Figures 2(c), 2(d), 3(c), and 3(d). The black line of MCPA is the cutting position of the segmented electrode $\left(L_{1}\right)$, as shown in Figure 3. It can be observed that, at the first mode, the strain of the continuous and segmented electrodes decreases monotonously from the fixed end to the free end in the strain contours of the MCPAs. However, at the second mode, there is a minimum strain magnitude at a certain region of the beam, which is much lower than at the fixed end, indicating the presence of a strain node.

\section{Results and Discussion}

4.1. Determination of Strain Nodes. Figure 4 shows the dependence of normalized deflection and normalized strain distribution of the MCPAs with continuous and segmented electrodes on the dimensionless position along the beam axis $\bar{x}=x / L$. Here, the normalized deflection curve is monotonically decreasing, and there is no zero point at the first mode, as shown in Figure 4(a). At the second mode there is a zero point on the normalized deflection curve, indicating the existence of strain nodes. In Figure 4(b), at the first mode, the strain distribution curve is monotonically increasing without strain node. At the second mode, there is a strain node at $\bar{x}=x / L=0.216$, which is similar to the reported result [11]. Therefore, the existence of strain nodes at the second mode is analyzed by both the theoretical and simulation models.

\subsection{Effect of Segmented Electrode Length on Tip Deflection.} To verify the influence of the segmented electrode length $L_{1}$ on the tip deflection of the MCPA, the $L_{1}$-tip deflection curves under different resonance frequency were simulated when $V=1-5 \mathrm{~V}, L_{1}=0-1.0 \mathrm{~mm}$, and $L=1 \mathrm{~mm}$, as shown in Figure 5. Figure 5(a) shows that tip deflection at the first mode does not change with the increase of $L_{1}$ under constant applied voltages. Figure 5(b) indicates that, at the second mode, as $L_{1}$ increases from 0 to $0.216 \mathrm{~mm}$, the tip deflection increases monotonically, but as $L_{1}$ increases from 0.216 to $1 \mathrm{~mm}$, the tip deflection decreases nonmonotonically. The tip deflection increases and reaches a peak at $L_{1}=0.216 \mathrm{~mm}$ (strain node); then the tip deflection decreases and reaches the lowest value at $L_{1}=0.620 \mathrm{~mm}$. Meanwhile, the maximum value of tip deflection increases as the applied voltage increases. At different modes, the electrode is segmented at the strain node and the electrodes are connected in different ways, in which the modal electromechanical coupling coefficient can reach a large value. For the modal displacement in the spatial potential, the cancelation is prevented to increase the tip deflection at the second mode. It indicates that the mechanism of the segmented electrode to avoid the modal displacement offsets at the high modes has been verified for the MCPAs. By adjusting the length of the segmented electrode and positioning segmentation location at the strain node, the larger tip deflection value can be obtained to improve the actuation performance.

4.3. Tip Deflections with the Segmented Electrode under Different Excitation Frequencies. Under different excitation frequencies, the tip deflection at the first and second modes of the MCPA with continuous and segmented electrodes is described in Figures 6 and 7. The tip deflection reaches a peak at the resonance frequency, and the peak value increases with the growth of the applied voltages, as shown in Figure 6. For continuous electrodes and segmented electrodes MCPAs, the tip deflections at the first mode are almost the same. At the second mode, the tip deflection of the MCPA-S at the second mode is almost $100 \%$ larger than that of the MCPA-C, as depicted in Figure 7. The maximum error of the tip deflection at the second mode is $6.8 \%$ between the theoretical and simulation results, and the theoretical calculation results of tip deflection are close to its simulation results. It reveals that, under different excitation frequencies, there is zero/one strain node at the first/ second mode for the MCPAs, which has different degrees of influence on the tip deflection. Furthermore, the series/parallel connection is valid for MCPA-S at the first/second mode. At the second mode, the dynamic strain distribution of the beam can change the strain direction on both sides of the strain node [11]. When the strain nodes are covered by the continuous electrodes, the tip deflection of the beam is canceled. Therefore, the optimized segmented electrode is cut at the strain node, and the wires of the segmented electrodes are connected in series at the first mode and in parallel at the second mode. It can prevent the modal displacement in the electric potential from canceling out [21] and improve the actuation performance of MCPA-S.

4.4. Dependence of Tip Deflection on Applied Voltage. To perceive the dependence of tip deflection on applied voltage, theoretical calculations and simulations are carried out on the tip deflection of MCPA-C and MCPA-S under different applied voltages. Here, the tip deflection increases linearly with the increase of the applied voltage from 1 to $5 \mathrm{~V}$, as indicated in Figure 8. The theoretical slope of the applied voltage-tip deflection curve at the first mode is $12.85 \mu \mathrm{m} / \mathrm{V}$ at $24 \mathrm{kHz}$ in Figure 8(a). In Figure 8(b), at $150 \mathrm{kHz}$, the theoretical slopes of the applied voltage-tip deflection curves at 
TABLE 4: Materials properties of the piezoelectric layer.

\begin{tabular}{lcc}
\hline Piezoelectric constant $d(\mathrm{pm} \mathrm{V}-1)$ & Dielectric constant & Elastic constant $(\mathrm{GPa})$ \\
\hline$d_{31}=-123$ & $\varepsilon_{11}=730 \varepsilon_{0}$ & $c_{11}=139$ \\
& & $c_{12}=77.8$ \\
$d_{33}=289$ & $\varepsilon_{33}=635 \varepsilon_{0}$ & $c_{13}=74.3$ \\
$d_{15}=496$ & & $c_{33}=115$ \\
& & $c_{44}=25.6$ \\
\hline
\end{tabular}

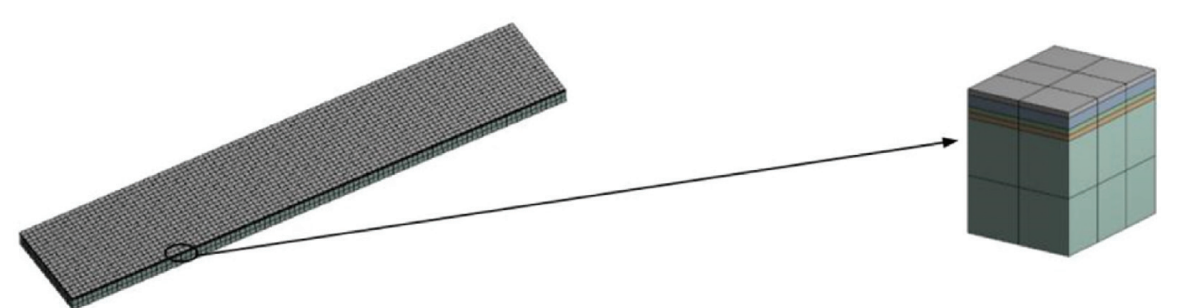

(a)

(b)

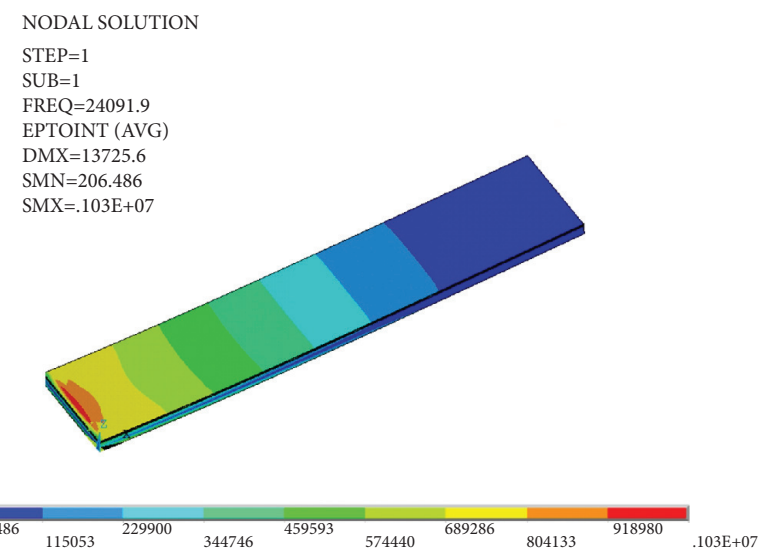

(c)

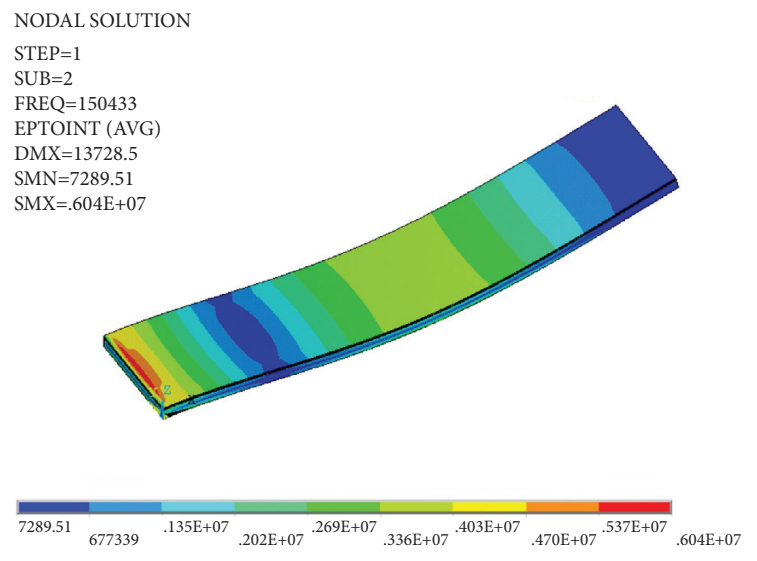

(d)

FIgURE 2: (a) The FEM mesh and (b) local view of the mesh at the strain node and the stress contours of the MCPA-C at the first (c) and (d) second mode.

the second mode are $0.88 \mu \mathrm{m} / \mathrm{V}$ and $1.73 \mu \mathrm{m} / \mathrm{V}$, respectively, for MCPA-C and MCPA-S. The theoretical and simulation tip deflections of the MCPA-S are 97\% larger than that of MCPA-C, and the maximum relative error is $7.8 \%$. Obviously, the theoretical values are in good agreement with the simulation values. It shows that the presented theoretical model can correctly estimate the dynamic tip deflection of the MCPA under the applied voltage.

4.5. Dependence of the Tip Deflection on the Beam Length. In order to investigate the relationship between the tip deflection and the beam length [18], the values of tip deflection of the MCPA were obtained when $L=0-2.0 \mathrm{~mm}$ and $V=1-5 \mathrm{~V}$. The theoretical/simulation results of the $L$-tip deflection curves of MCPA-C and MCPA-S at first and second mode are given in Figures 9 and 10. Under different constant voltages, as the beam length $L$ increases from 0 to $2 \mathrm{~mm}$, the tip deflections at the first and second modes increase nonlinearly, which is consistent with [22]. At the second mode, the tip deflection of the segmented electrode is obviously greater than that of the continuous electrode, as shown in Figures 10(a) and 10(b). By analyzing the relative deviations of the tip deflection when $V=1 \mathrm{~V}$ (low applied voltage), the theoretical and simulation results are expressed by lines and dots, respectively, as shown in Figure 10(c). The maximum deviations of the tip deflections between the theoretical and simulation results are $4.9 \%$ for the MCPA-S and 4.1\% for the MCPA-C. It is similar to the reported results [21]. The validity of the proposed model has been verified for segmented electrode MCPA. Therefore, it is a useful strategy to improve the actuation performance by adjusting the beam length $L$, which can be used to drive microelectromechanical switches [3].

4.6. Dependence of Tip Deflection on the Substrate/Piezoelectric Layer Thickness Ratio. In this section, $r=E_{s} / E_{\mathrm{p}}$ and $h=h_{\mathrm{s}} / h_{\mathrm{p}}$ are defined to describe Young's modulus ratio 


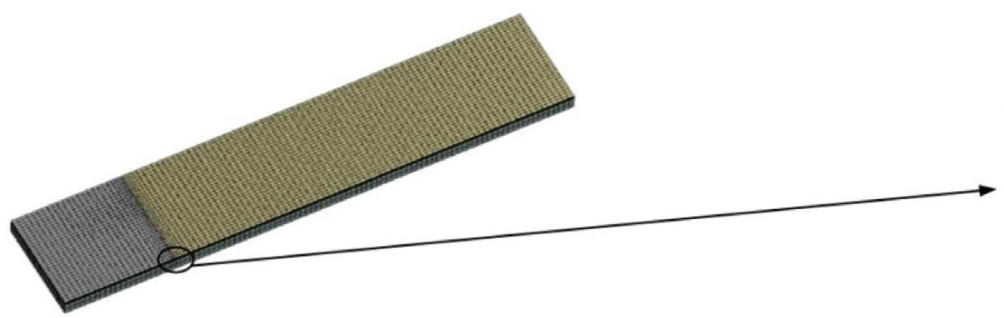

(a)

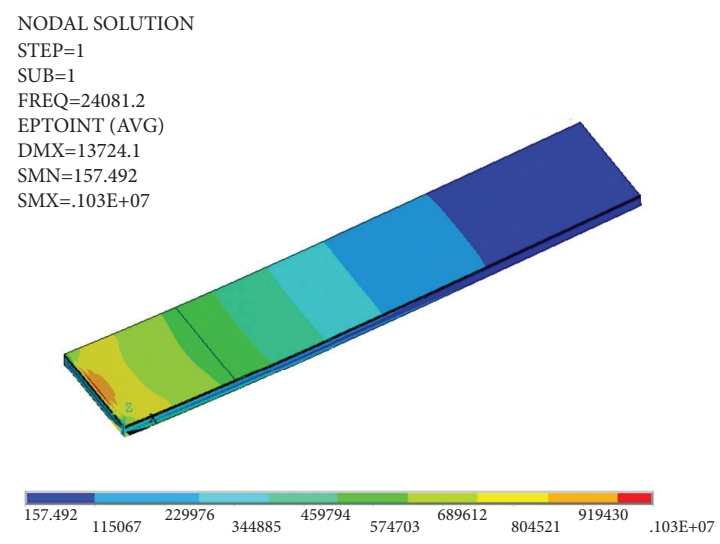

(c)

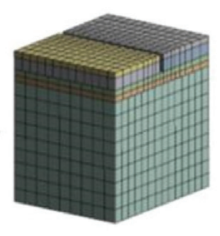

(b)

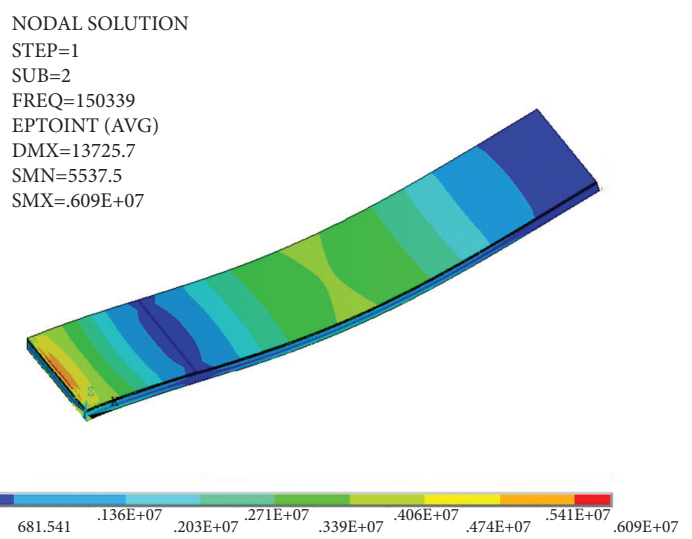

(d)

Figure 3: (a) The FEM mesh and (b) local view of the mesh at the strain node and the stress contours of the MCPA-S at the first (c) and (d) second mode.

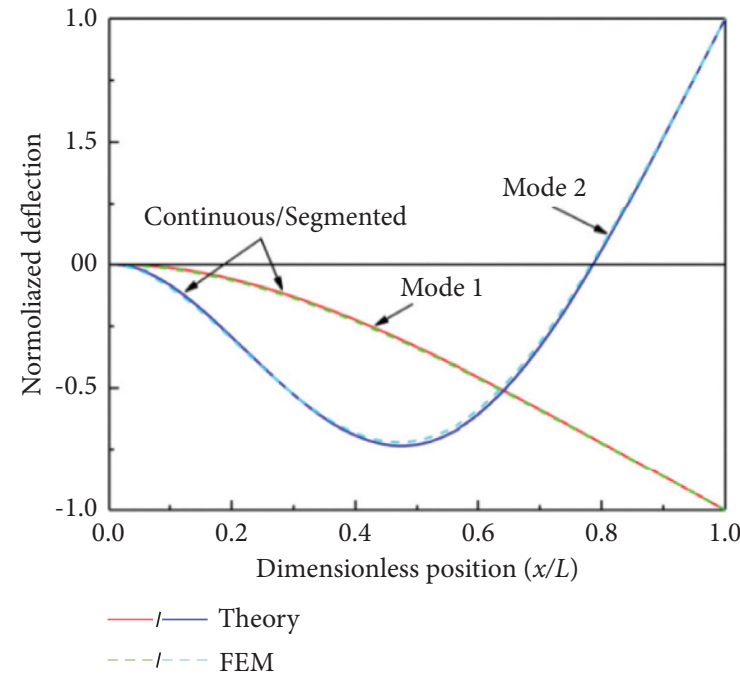

(a)

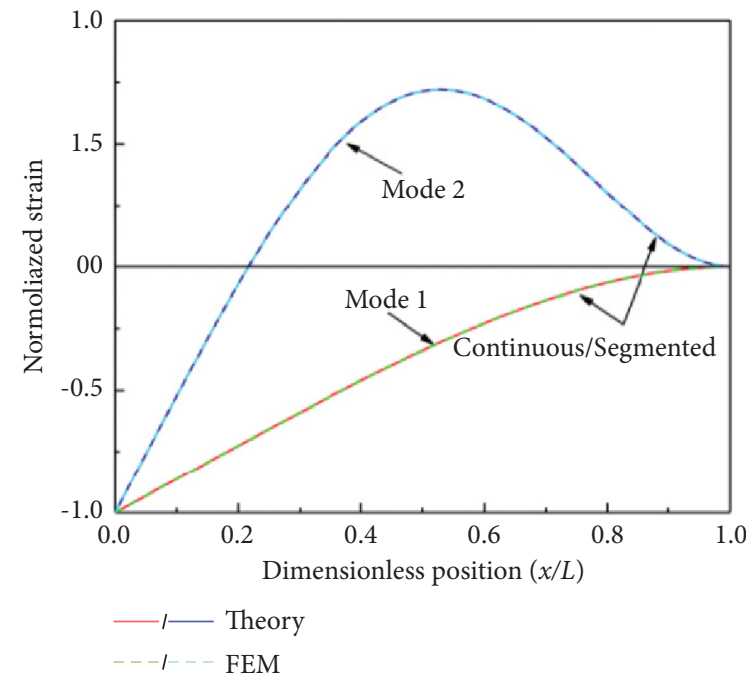

(b)

FIgURE 4: The (a) normalized deflection and (b) normalized strain distribution of the first two modes are simulated by MATLAB and FEM.

and thickness ratio between the substrate and the piezoelectric layer [23]. In order to investigate dependence of tip deflection on the thickness ratio between the substrate and piezoelectric layer, when $V=1 \mathrm{~V}, h_{e}=1 \mu \mathrm{m}$, $L=1 \mathrm{~mm}, b=200 \mu \mathrm{m}$, and $h_{i}=1 \mu \mathrm{m}$, the $h$-tip deflection curves of MCPAs at the first two modes under different
Young's modulus ratios were simulated and analyzed. They are shown in Figures 11 and 12. Two monotonously changing regions are formed on two sides of the maximum point of each curve. At the first and second modes, as $h$ and $r$ increase, the tip deflections increase/decrease in the upward/downward region. In particular, when the 


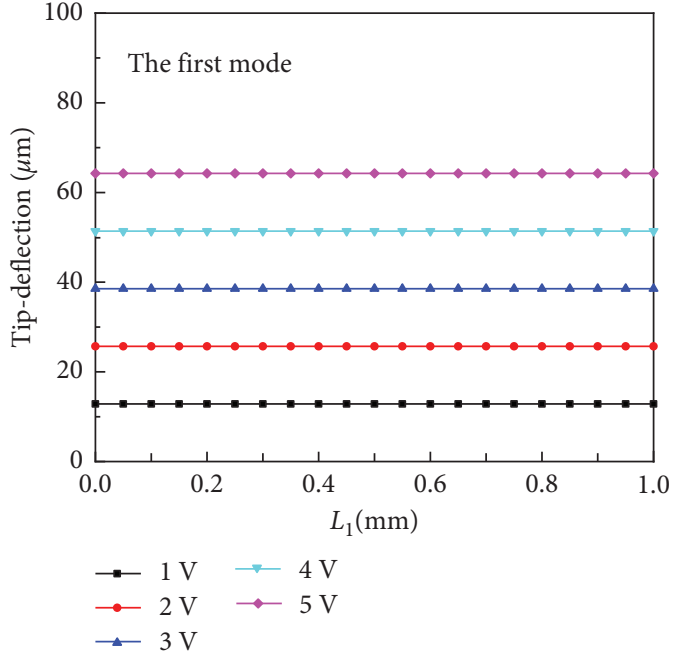

(a)

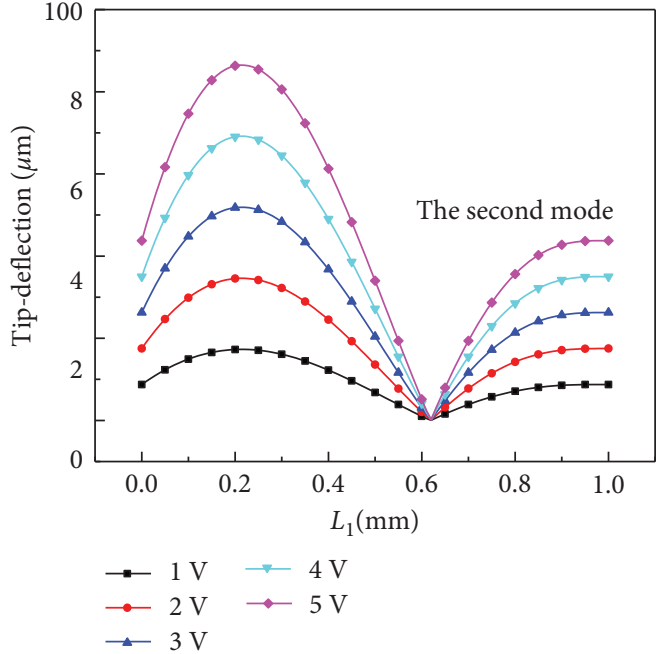

(b)

FiguRE 5: Effect of the segmented electrode length $L_{1}$ on tip deflection for the first (a) and second (b) modes under different applied voltages.

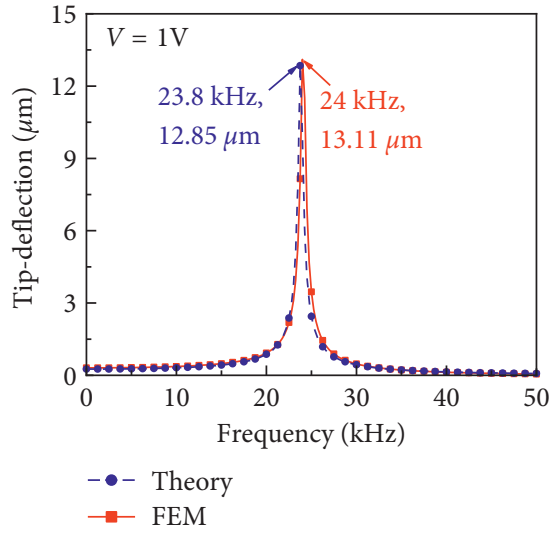

(a)

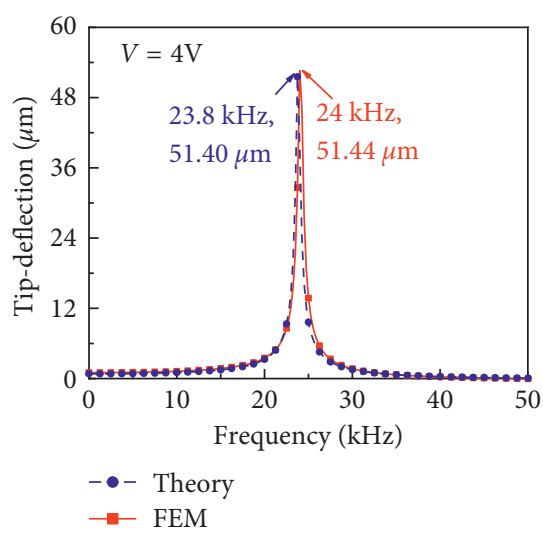

(d)

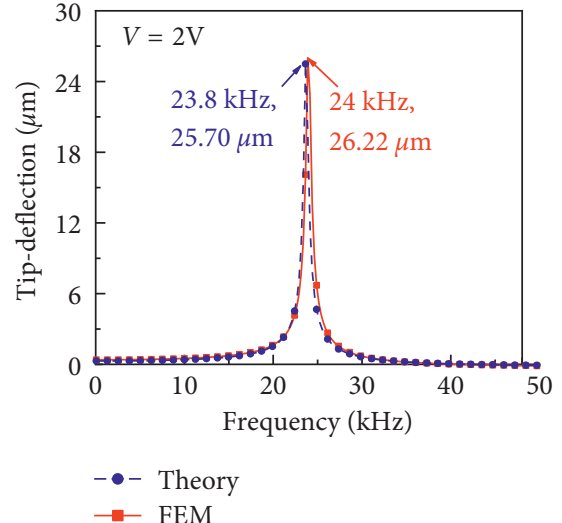

(b)

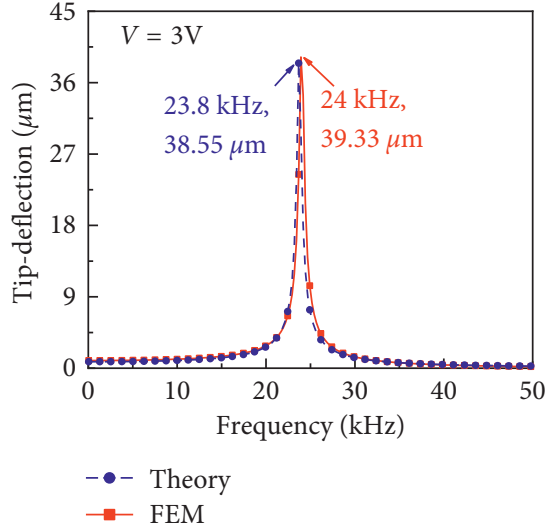

(c)

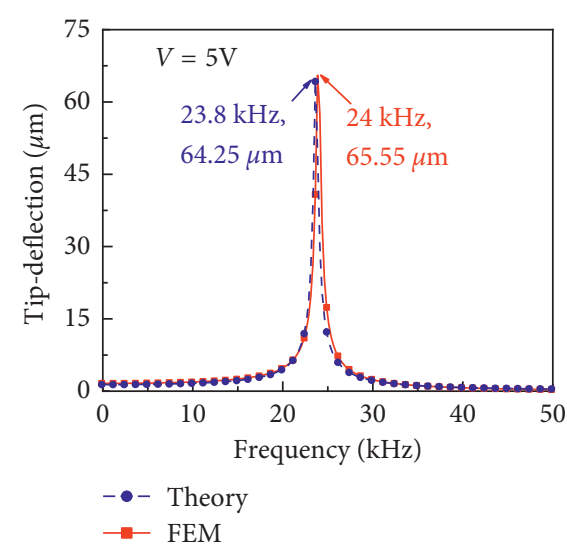

(e)

Figure 6: Tip deflection of the MCPA with the continuous and segmented electrodes for the first mode under different excitation frequencies at (a) $V=1 \mathrm{~V}$, (b) $V=2 \mathrm{~V}$, (c) $V=3 \mathrm{~V}$, (d) $V=4 \mathrm{~V}$, and (e) $V=5 \mathrm{~V}$.

thickness ratio is constant $(h=0-2.3)$, the tip deflection increases with the increase of Young's modulus $(r=0.8-2.4)$. In the downward region, when $h$ is constant
( $h=2.3-5)$, the tip deflection decreases as $r$ increases $(r=0.8-2.4)$. For MCPAs at the first and second mode, the change tendencies of tip deflections with $h$ and $r$ are 


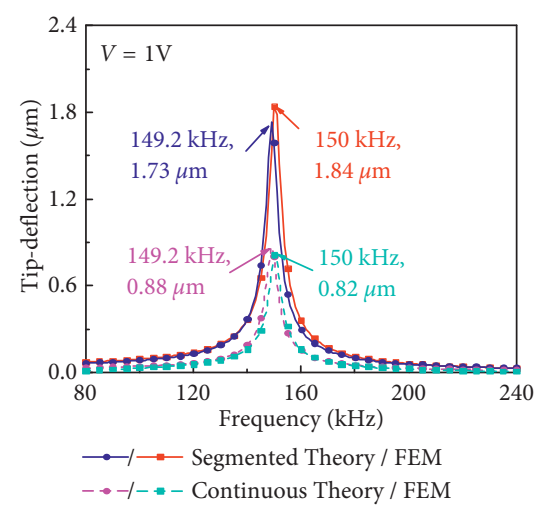

(a)

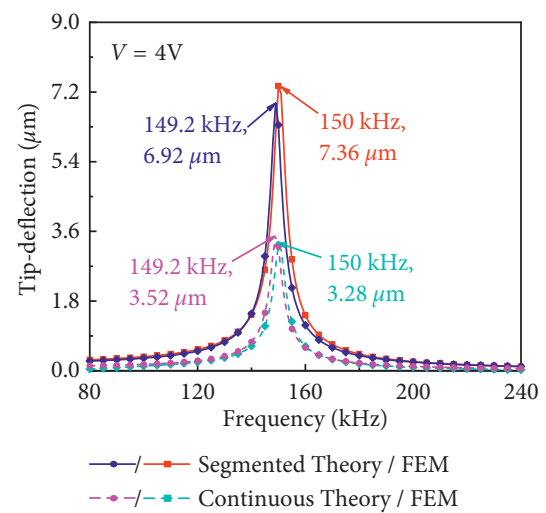

(d)

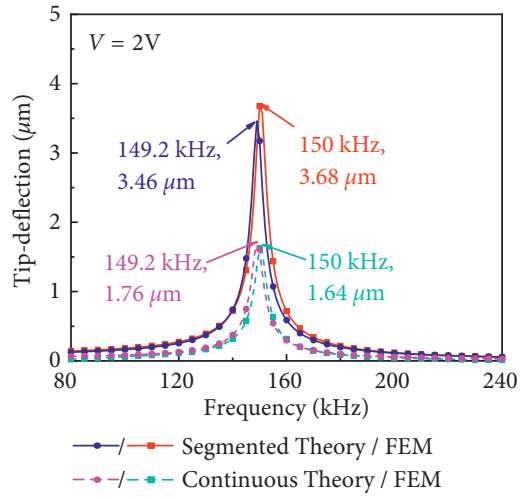

(b)

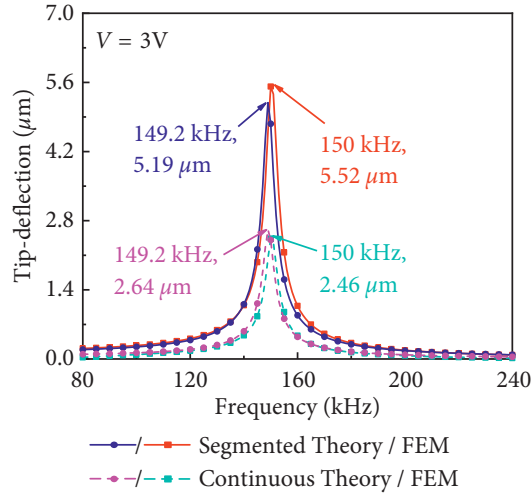

(c)

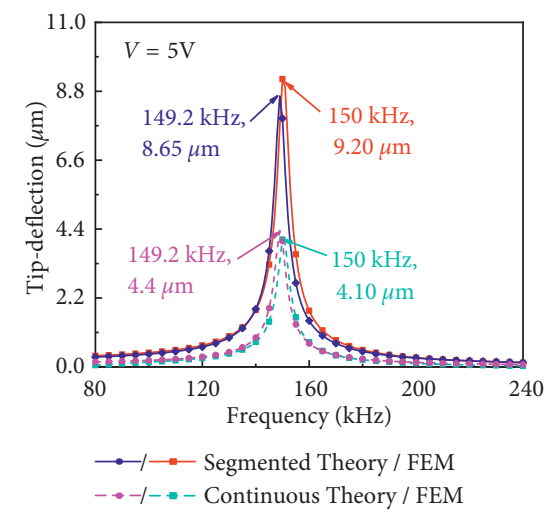

(e)

Figure 7: Harmonic response of the MCPA with the continuous and segmented electrodes for the second mode at (a) $V=1 \mathrm{~V}$, (b) $V=2 \mathrm{~V}$, (c) $V=3 \mathrm{~V}$, (d) $V=4 \mathrm{~V}$, and (e) $V=5 \mathrm{~V}$.

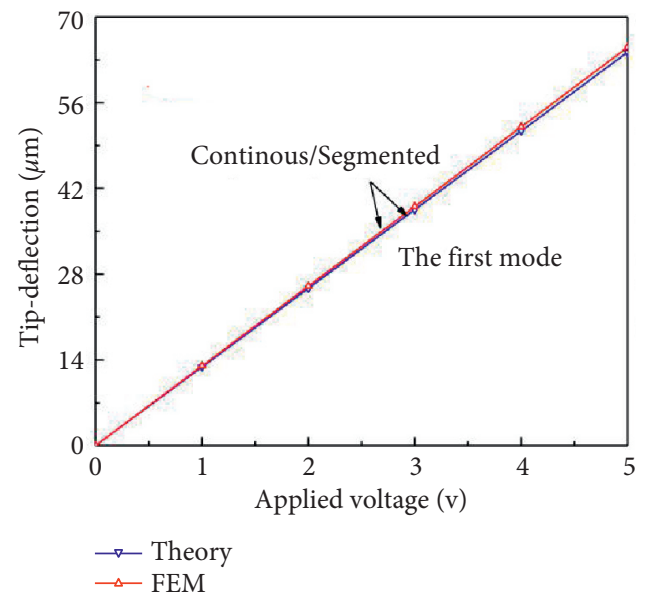

(a)

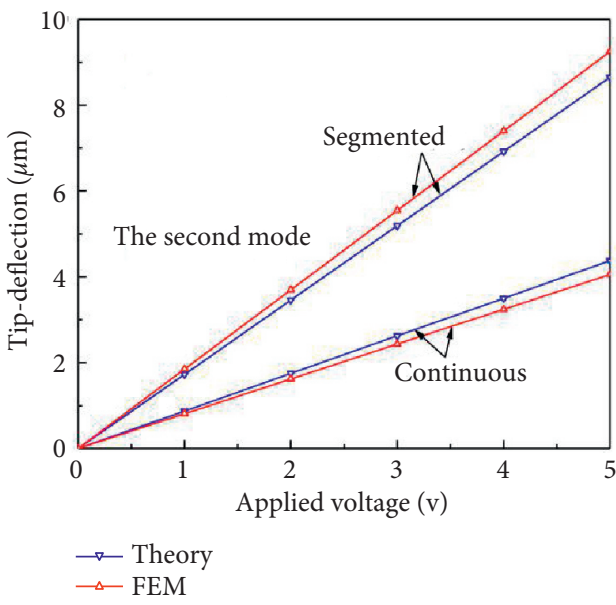

(b)

Figure 8: Dependence of tip deflection on applied voltage for the $L=2 \mathrm{~mm}$ beam at $24 \mathrm{kHz}$ for the first mode (a) and at $150 \mathrm{kHz}$ for the second mode (b).

similar through theoretical calculation and simulation. The tip deflection of MCPA-S is good with that of MCPA$\mathrm{C}$ at the first mode. Obviously, with a smaller thickness ratio and a larger Young's modulus ratio, the curve in the upward region has a larger slope; i.e., in this region the tip deflection is more sensitive to the thickness change, which is beneficial for position sensing [24]. At the second mode, the slopes of the tip deflection curves for MCPA-S are significantly larger than that of the curves for MCPA-C, as shown in Figure 12. It indicates that the tip deflection of MCPA-S is more sensitive to change of thickness ratio and Young's modulus ratio. A smaller 


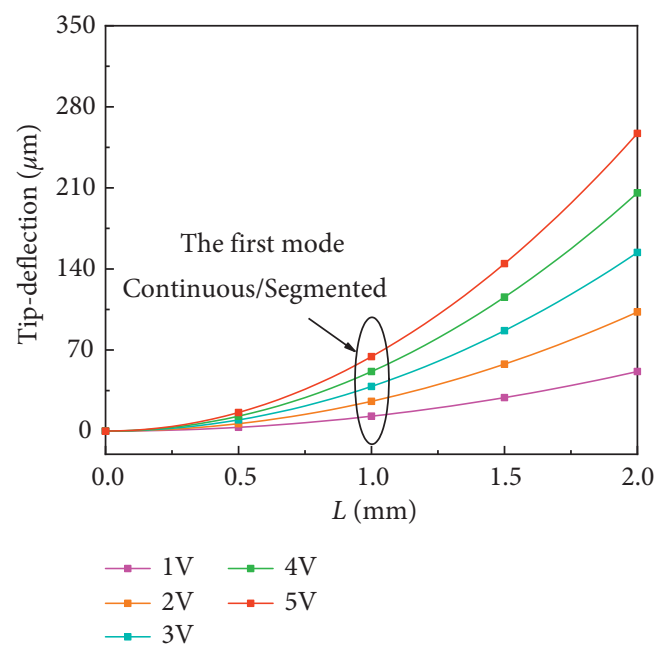

FIgURE 9: Theoretical and FEM simulation results of the dependence of tip deflection on beam length $L$ at the first mode at $V=1-5 \mathrm{~V}$.

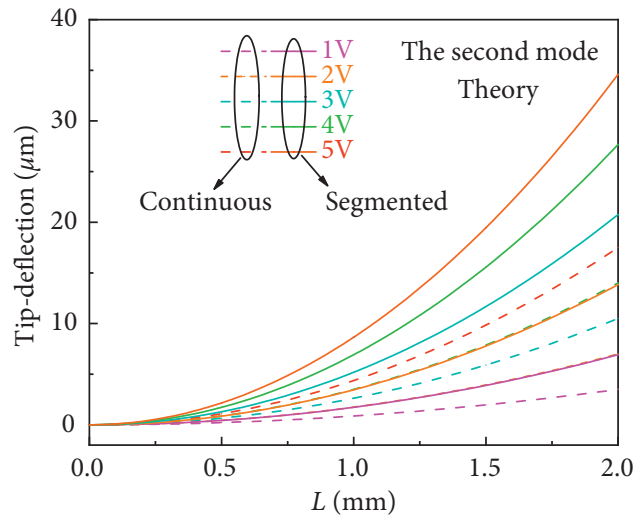

(a)

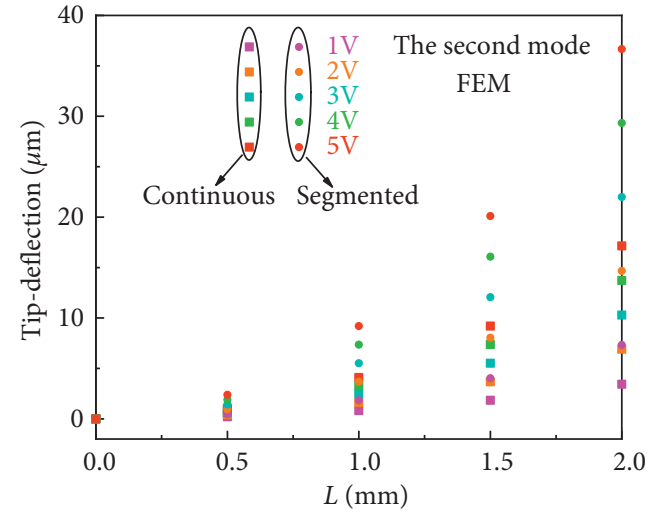

(b)

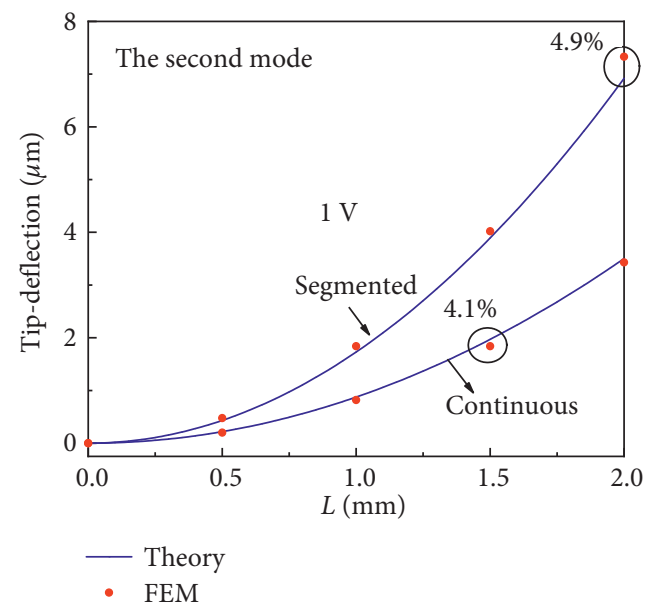

(c)

Figure 10: Theoretical (a) and FEM simulation (b) results of the dependence of tip deflection on beam length $L$ at the second mode at $V=1-5 \mathrm{~V}$ and the theoretical and FEM simulation results at the second mode at $V=1 \mathrm{~V}$ (c). 


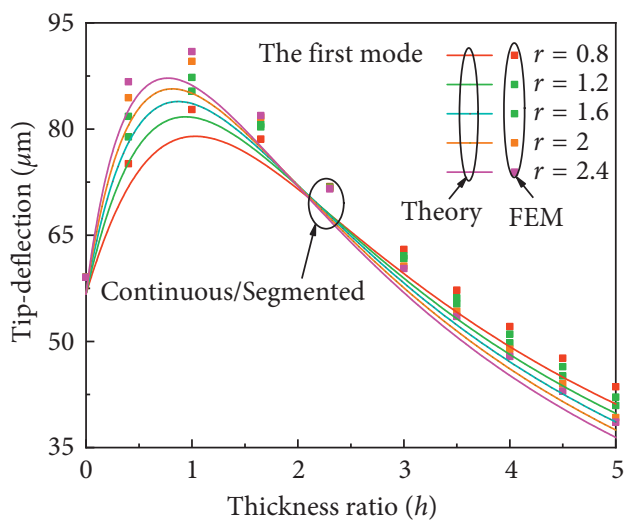

FIGURE 11: Theoretical and FE simulation results of the dependence of tip deflection on thickness ratio $h$ at the first mode under different Young's modulus ratios $r$.

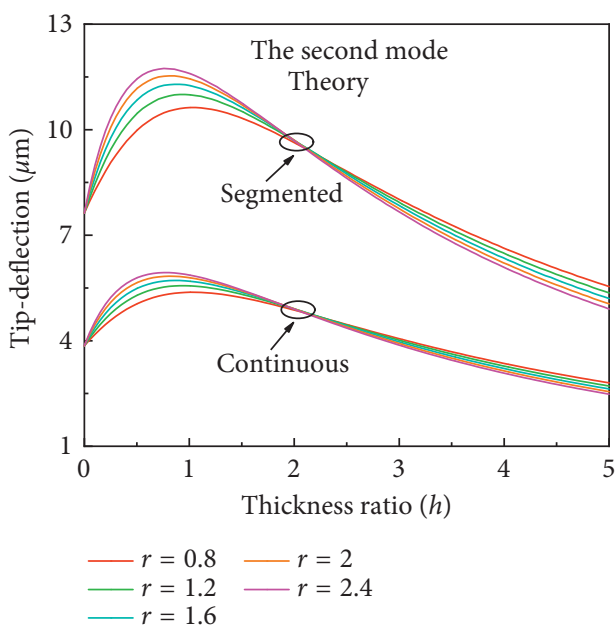

(a)

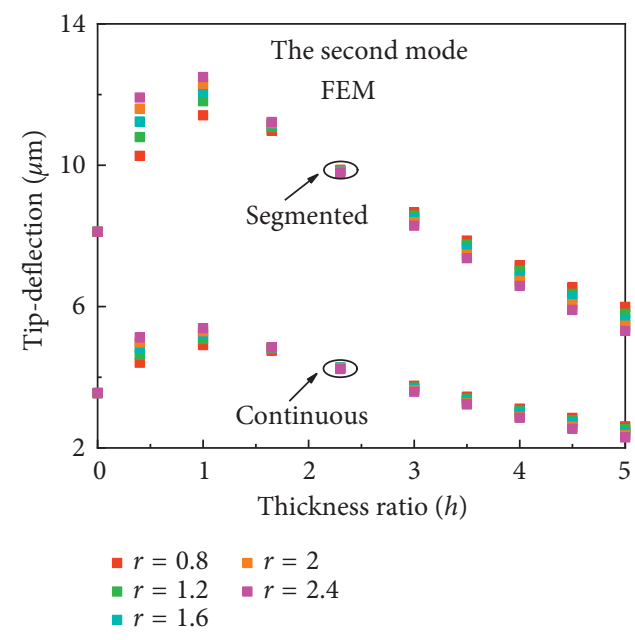

(b)

Figure 12: Theoretical (a) and FE simulation (b) results of the dependence of tip deflection on thickness ratio $h$ at the second mode under different Young's modulus ratios $r$.

thickness ratio and larger Young's modulus ratio can achieve the maximum tip deflection, thereby improving the actuation performance of the MCPAs [25].

\section{Conclusions}

In summary, by considering the influence of the strain node on the beam at the high modes for the MCPAs, the optimal position of segmented electrode should be at the strain node. At the high modes of MCPAs, the tip deflections of the MCPA-S are larger than that of the MCPA-C, so the actuation performance can be improved. The strain node of the MCPA-S is $0.216 \mathrm{~mm}$ at the second mode, and the developed complete electromechanical coupling model can predict the tip deflection at the first two modes. The results of theoretical calculation and simulation indicate that the tip deflections of the MCPA-S are consistent with the MCPA-C at the first mode. The tip deflection of the MCPA-S is almost $100 \%$ larger than that of the MCPA-C at the second mode, and the tip deflection at the second mode has a maximum error of
$6.8 \%$ between the theoretical and simulation results. Obviously, the theoretical values and the simulation results are relatively close, indicating that the proposed model can precisely estimate the tip deflection of MCPAs. The reliance of tip deflection on segmented electrode length, actuation frequency, voltage, beam length, substrate/piezoelectric thickness, and Young's modulus ratio are also discussed. The results indicate that, for the MCPA-S, under a certain beam length and high voltage pressure, a smaller thickness ratio and a larger Young's modulus ratio of the substrate/piezoelectric layer are beneficial to gain a larger tip deflection. The proposed model verifies the mechanism of MCPA-S to avoid the modal displacement offsets at the high modes, and predicted results can provide valuable guidance for optimizing the construction and efficiency of MCPAs.

\section{Data Availability}

The data that support the findings of this study are available from the corresponding author upon reasonable request. 


\section{Conflicts of Interest}

The authors declare that there are no conflicts of interest with respect to the research, authorship, and/or publication of this article.

\section{Acknowledgments}

This work was supported by NNSF of China (11832016 and 51775471), Hunan Innovative Province Construction Special Major Landmark Innovation Demonstration Project, Changsha Zhuzhou Xiangtan Landmark Engineering Technology Project (2019XK2303 and 2020GK2014), Hefei General Machinery Research Institute Co., Ltd Project (2021ZKKF043), and Postgraduate Scientific Research Innovation Project of Hunan Province (CX20200642).

\section{References}

[1] Z. Xu, Z. Yang, K. Wang et al., "A bionic inertial piezoelectric actuator with improved frequency bandwidth," Mechanical Systems and Signal Processing, vol. 156, Article ID 107620, 2021.

[2] S. J. Behrouz, O. Rahmani, and S. A. Hosseini, "On nonlinear forced vibration of nano cantilever-based biosensor via couple stress theory," Mechanical Systems and Signal Processing, vol. 128, no. 3, pp. 19-36, 2019.

[3] Y. B. Chen and Z. Yan, "Investigation of pull-in behaviors of a nanoswitch tuned by piezoelectric and flexoelectric effects," International Journal of Mechanical Sciences, Article ID 107620, 2019.

[4] Q. F. Lü, X. F. Wang, K. Lu, and R. H. Huan, "Nonlinear stochastic optimal control using piezoelectric stack inertial actuator," Shock and Vibration, vol. 7, Article ID 5372045, 2020.

[5] R. F. Wang, L. Wang, J. M. Jin, B. T. Jia, Q. Zhang, and D. W. Wu, "Excitation method and electromechanical coupling dynamic model of a novel torsional piezoelectric actuator," Mechanical Systems and Signal Processing, vol. 154, Article ID 10758, 2021.

[6] V. Zhang, M. Faucher, D. Theron, C. Morelle, and L. Buchaillot, "Improved analytical modelling and finite element verification of stressed $\mathrm{GaN}$ microbeam resonators by piezoelectric actuation," Journal of Micromechanics and Microengineering, vol. 27, no. 9, Article ID 095001, 2017.

[7] Y. Zhang, T.-F. Lu, and S. Al-Sarawi, "Formulation of a simple distributed-parameter model of multilayer piezoelectric actuators," Journal of Intelligent Material Systems and Structures, vol. 27, no. 11, pp. 1485-1491, 2015.

[8] S. M. Afonin, "Structural-parametric model multilayer electromagnetoelastic actuator for nanomechatronics," International Journal of Physics, vol. 7, no. 2, pp. 50-57, 2019.

[9] P. Shivashankar and S. Gopalakrishnan, "Design, modeling and testing of d33-mode surface-bondable multilayer piezoelectric actuator," Smart Materials and Structures, vol. 29, pp. 0-16, 2020.

[10] S. Peng, X Zheng, J. Sun et al., "Modeling of a micro-cantilevered piezo-actuator considering the buffer layer and electrodes," Journal of Micromechanics and Microengineering, vol. 22, no. 6, pp. 561-566, 2012.

[11] A. Erturk and D. J. Inman, "A distributed parameter electromechanical model for cantilevered piezoelectric energy harvesters," Journal of Vibration and Acoustics, vol. 130, no. 4, Article ID 041002, 2008.

[12] A. Erturk and D. J. Inman, "On mechanical modeling of cantilevered piezoelectric vibration energy harvesters," Journal of Intelligent Material Systems and Structures, vol. 19, no. 11, pp. 1311-1325, 2008.

[13] R. Ly, M. Rguiti, S. D’Astorg, A. Hajjaji, C. Courtois, and A. Leriche, "Modeling and characterization of piezoelectric cantilever bending sensor for energy harvesting," Sensors and Actuators A: Physical, vol. 168, no. 1, pp. 95-100, 2011.

[14] A. Erturk, P. A. Tarazaga, and J. R. Farmer, "Effect of strain nodes and electrode configuration on piezoelectric energy harvesting from cantilevered beams," Journal of Vibration and Acoustics, vol. 131, no. 1, pp. 0110101-0110111, 2009.

[15] D. Zizys, R. Gaidys, R. Dauksevicius, V. Ostasevicius, and V. Daniulaitis, "Segmentation of a vibro-shock cantilever-type piezoelectric energy harvester operating in higher transverse vibration modes," Sensors, vol. 16, no. 1, pp. 1-14, 2016.

[16] S. Rafique and S. A. Shah, "Analysis over improving the power out of a piezoelectric energy harvester using segmented electrodes," Mehran University Research Journal of Engineering and Technology, vol. 36, no. 1, pp. 177-182, 2017.

[17] Y. Liu, X. Yang, W. Chen, and D. Xu, "A bonded-type piezoelectric actuator using the first and second bending vibration modes," IEEE Transactions on Industrial Electronics, vol. 63 , no. 3, pp. 1676-1683, 2016.

[18] D. Bath and A. Salehian, "A novel 3D folded zigzag piezoelectric energy harvester; modeling and experiments," Smart Materials and Structures, vol. 28, Article ID 025011, 2019.

[19] J. S. Yang and H. Y. Fang, "Analysis of a rotating elastic beam with piezoelectric films as an angular rate sensor," IEEE Transactions on Ultrasonics, Ferroelectrics, and Frequency Control, vol. 49, no. 6, pp. 798-804, 2002.

[20] M. Brissaud, S. Ledren, and P. Gonnard, "Modelling of a cantilever non-symmetric piezoelectric bimorph," Journal of Micromechanics and Microengineering, vol. 13, no. 6, pp. 832-844, 2003.

[21] O. Bilgen, A. Erturk, and D. J. Inman, "Analytical and experimental characterization of macro-fiber composite actuated thin clamped-free unimorph benders," Journal of Vibration and Acoustics, vol. 132, no. 5, Article ID 051005, 2010.

[22] J. C. Doll, B. C. Petzold, B. Ninan, R. Mullapudi, and B. L. Pruitt, "Aluminum nitride on titanium for CMOS compatible piezoelectric transducers," Journal of Micromechanics and Microengineering: Structures, Devices, and Systems, vol. 20, no. 2, Article ID 25008, 2010.

[23] H. A. Tinoco and A. L. Serpa, "Voltage relations for debonding detection of piezoelectric sensors with segmented electrode," Mechanical Systems and Signal Processing, vol. 31, pp. 258-267, 2012.

[24] X. Cheng, X. L. Sun, Y. Liu et al., "Integrated optoelectronic position sensor for scanning micromirrors," Sensors, vol. 18, no. 4, Article ID 982, 2018.

[25] J. Shen, H. Wang, and S. Zheng, "Size-dependent pull-in analysis of a composite laminated micro-beam actuated by electrostatic and piezoelectric forces: generalized differential quadrature method," International Journal of Mechanical Sciences, vol. 135, pp. 353-361, 2018. 